\title{
Innerstaatlicher Rechtsschutz für internationale Bedienstete?
}

\author{
Von Dr. iur. utr. habil. Ludwig Gramlich, Würzburg
}

„Keine Auffangzuständigkeit deutscher Gerichte nach Art. 19 IV GG - beschränkte Kontrolle primären Staatenverbandsrechts - aber: Überprüfung des Handelns des Staatenvertreters im Hauptorgan Internationaler Organisationen allseitige Grundgesetzbindung, ausgestaltet durch Grundrechte und Völkerrechtsfreundlichkeit - vorbeugender Rechtsschutz durch Verwaltungsgerichte bzw. BVerfG - Bedeutung für Vermögensschutz und für Kommunikationsbereich internationaler Bediensteter".

I. Problemaufri $\beta$

Eine ansehnliche Zahl internationaler Bediensteter sieht sich seit kurzem nicht unerheblichen Reallohneinbußen ausgesetzt, die ihnen von hoher Verbandshand verordnet wurden ${ }^{1}$. Es bedarf wohl keiner weiteren Darlegungen, daß derartige Maßnahmen nicht gerade von ungeteiltem Beifall der Betroffenen begleitet werden. Nachdem freilich Arbeitskampf, insbesondere Streik zur Abwendung bzw. Aufhebung dieser Belastungen zwar kaum je einmal als verbandsrechtswidrige Handlungen ausdrücklich verboten sind ${ }^{2}$, die Praxis bei den verschiedenen intergouvernementalen Dienstherrn aber ebensowenig eindeutig die Legalität solchen Verhaltens zu belegen vermag ${ }^{3}$, bleibt die Frage nach der Möglichkeit auch eines staatlichen gerichtlichen Schutzes überaus aktuell, ja vorrangig. Denn bislang existieren kaum institutionelle Vorkehrungen, die den zwischenstaatlichen Bediensteten eine Mitwirkung oder gar Mitbestimmung bei Entscheidungen des zuständigen Verbandsorgans über dienst-, insbesondere besoldungsrechtliche Gegenstände einräumen ${ }^{4}$.

\section{Einzelfragen im prozessualen Bereich}

1. Dabei begegnet freilich der Versuch, nationale, mitgliedstaatliche Gerichte mit dergleichen Dienstrechtsangelegenheiten befassen zu wollen, hierzulande auf den ersten Blick scheinbar unüberwindlichen Hindernissen. Zumindest für die künftige judizielle Praxis haben nämlich für den zahlenmäßig weitaus überwiegenden Teil der Verbandsbediensteten, deren Beschäftigungsverhältnisse nicht dem nationalen Arbeitsrecht (des Dienstorts) unterliegen ${ }^{5}$, die beiden „Eurocontrol“-Entscheidungen des Bundesverfassungsgerichts ${ }^{6}$, vornehmlich das zweite Erkenntnis vom 10.11.1981, wohl endgültig klargestellt, daß weder Art. 19 IV GG noch das Rechtsstaatsprinzip eine Art „Auffangzuständigkeit“ deutscher Gerichte begründeten, wenn im Einzelfall eine internationale deutsche Jurisdiktion ${ }^{7}$ nicht gegeben $\operatorname{sei}^{8}$. Wesentlich erscheine allein, ob eine zwischenstaatliche Einrich- tung durch einen wirksamen völkerrechtlichen Akt geschaffen worden sei und sich diese bei dem je im Streit befindlichen Verbandsverhalten nicht völlig von dieser international-rechtlichen Grundlage gelöst habe? ${ }^{9}$. Wenn sohin Verbandsorgane in bezug auf die Rechtsverhältnisse der Organisationsbediensteten normierend wie auch vollziehend tätig werden, so üb(t)en sie keine deutsche öffentliche Gewalt aus. Nicht (mehr) bedeutsam ist mithin, daß die Verbandserrichtung nur im Wege eines "Gesamtakts" ${ }^{10}$ aller Mitgliedstaaten stattfinden konnte und hierbei auch die Bundesrepublik Deutschland iure imperii beteiligt war.

2. Letztlich vermag die Begründung einer exklusiven Zuständigkeit der verbandsinternen Instanzen aber nur dann eine Rechtsschutzgewährung durch deutsche (Verwaltungs- $)^{11}$ Gerichte zu hindern, wenn solches durch

${ }^{1}$ Einiges hierzu bei Gramlich, ZBR 1985, 37 (38); s. a. Ried, ZBR 1984, 330 (334 f.). Zu Beginn des Jahres 1985 waren in dieser Sache vor mehreren internationalen Verwaltungsgerichten, darunter auch dem EuGH, Klagen betroffener Bediensteter anhängig.

2 Übersicht bei Plantey, Droit et pratique de la fonction publique internationale, 1977, 150 f.; s. ferner M.Schröder, ZBR 1984, 1 (2 ff.).

3 Plantey, aaO, $152 \mathrm{ff}$; vgl. auch H. J. Hahn, Einführung, in: Kaiser/Mayer/Ule (Hrsg.), Recht und System des öffentlichen Dienstes, Bd. 4, 1973, 25 (49 f.). Offengelassen wurde die Antwort bsp. im Erkenntnis No. 39 des O.E.C.D. Appeals Board vom 27.7.1965 (Domergue), wo ein Streik von Organisationsbediensteten am 11./12.2. jenes Jahres zur Debatte stand (s. Commission de recours de l'OECE/OCDE, Recueil des décisions 1 à 62, 1979, 110 [111]), ebenso in der EuGH-Entscheidung über die verb. Rs. 44, 46, 49774 vom 18.3.1975 (Slg. 1975, $383[395])$

${ }_{4}^{4}$ Dem soll allerdings nunmehr in der Europäischen Weltraum-Organisation, der „European Space Agency“, abgeholfen werden, unter ausdrücklicher Bezugnahme auf mitgliedstaatliche Rechtsentwicklungen, etwa das Bundespersonalvertretungsgesetz vom 15.3.1974. Vgl. auch die Û̉bersicht bei Ruzié, La protection des agents internationaux, Colloque d'Aix-en-Provence der S. F.D.I. 1984, Typoskr. $8 \mathrm{ff}$.

${ }_{5}$ Hierzu Plantey, aaO, $36 \mathrm{ff}$, sowie z.B. das Erkenntnis des italienischen Kassationshofs n. 2054 vom 5.6.1976, Riv. Dir. Int. 49 (1976) 824 ff.

6 BVerfGE 58, $1 \mathrm{ff}$, mit Anm. von Gramlich, JZ 1982, $149 \mathrm{f}$; BVerfGE 59 , 63 ff., mit Anm. von Busch, DVBl. 1982, 579 f., und von Gramlich, DöV 1982, $407 \mathrm{ff}$. Eingehend dazu auch Schwarze, EuGRZ 1983, 117 (118ff.).

${ }_{7} \mathrm{Zu}$ dieser Sachentscheidungsvoraussetzung s. hier den in "Eurocontrol $\mathrm{II}^{\circ}$ angefochtenen Akt deutscher öffentlicher Gewalt i. S.v. Art.93I Nr.4a GG, nämlich den Beschluß des VGH Mannheim, DVBI. 1980, $127 \mathrm{f}$., mit Anm. von Gramlich, ebd., 459 f.; ferner BVerfGE 64, 1 (18 f.), sowie Juenger, Mich. L. Rev. 82 (1983/84) 1195 (1204).

${ }^{8}$ BVerfGE 58, 30; 59, 88; vgl. auch T.Stein, ZaöRV 42 (1982) 596 (597 f.), sowie BVerfGE 63, 343 (377f.).

9 BVerfGE 58, 29; 59, 85; ebenso bereits in bezug auf das (sekundäre) Recht der E. W. G. BVerfGE 22, 293 (296 ff.).

10 Vgl. Tomuschat, Art. 24 Abs. 1 (Zweitbearb. 1981), im Bonner Kommentar zum GG, 1950 ff., Rdn.16 (20); Stern, Das Staatsrecht der Bundesrepublik Deutschland I, 2. Aufl. 1984, 523 f. mit N. 44.

11 Seidl-Hohenveldern (Das Recht der Internationalen Organisationen einschließlich der Supranationalen Gemeinschaften, 4. Aufl. 1984, 193 [Rdn. 1351 a]) spricht allzu generell von Arbeitsgerichten. S. demgegenüber die von Schreuer, Die Behandlung internationaler Organakte durch staatliche Gerichte, 1977, $282 \mathrm{ff}$., angeführten Judikate, und ferner das bei Lachaume genannte Erkenntnis des französischen Kassationshofs vom 24.5.1978 (A.F.D.I. 25 [1979] 853 [893 f.]). 
wirksame innerstaatliche Gesetzgebung ausgeschlossen wurde ${ }^{12}$. Insofern legte das Bundesverfassungsgericht im zweiten „Eurocontrol“-Beschluß an die im Hinblick auf die Organisationsgerichtsbarkeit ergangene landesrechtliche Geltungsanordnung, das Vertragsgesetz, als Maßstab allein „fundamentale Grundsätze des Verfassungsrechts der Bundesrepublik " an $^{13}$ und erachtete es insbesondere nicht für bedeutsam, ob im Primärrecht einer Internationalen Organisation die Errichtung verbandsinterner "Administrative Tribunals" eigens vorgesehen sei $^{14}$; auch bei Fehlen ausdrücklicher Ermächtigungsvorschriften werde gemeinhin den zwischenstaatlichen Verbänden dieses Recht zuerkannt ${ }^{15}$.

Der Zweite Senat konnte nun vom Verwaltungsgericht der Internationalen Arbeitsorganisation (I. L. O.) angesichts dessen langjähriger, mehr als ein halbes Jahrhundert andauernder Aktivitäten ${ }^{16}$ leicht sagen, „Status und Verfahrensgrundsätze ... entsprechen ... einem internationalen Mindeststandard an elementarer Verfahrensgerechtigkeit ... und widersprechen ferner insgesamt auch nicht rechtsstaatlichen Mindestanforderungen im Sinne des Grundgesetzes" "17. Zumindest der erste Teil dieser Feststellung richtet sich dabei ersichtlich an früheren Erkenntnissen hinsichtlich „allgemeiner Regeln des Völkerrechts " im Sinne von Art. 25 GG aus, insbesondere zu fremdenrechtlichen Normen ${ }^{18}$; das Bundesverfassungsgericht nimmt offenbar das Bestehen eines generellen Selektionsmechanismus an, der gleichermaßen bei fremdstaatlichen wie intergouvernementalen (richterlichen) Akten wirkt und von Verfassungsrechts wegen anerkennungsfähige von nicht (mehr) akzeptablen Erkenntnissen zu scheiden nötigt ${ }^{19}$. Derartige Kennzeichnungen treffen aber ebenso auch andere Instanzen jüngeren Entstehungsdatums, ob sie nun explizit als "Administrative Tribunals" eingerichtet werden ${ }^{20}$ oder doch materiell richterliche Funktionen wahrnehmen ${ }^{21}$. Sie entscheiden regelmäßig wie ihr Genfer Pendant ,aufgrund rechtlich festgelegter Kompetenzen und zufolge eines rechtlich geordneten Verfahrens ausschließlich nach Maßgabe von Rechtsnormen und Rechtsgrundsätzen die (ihnen) unterbreiteten Verfahrensgegenstände ...; (ihre) Richter sind zur Unabhängigkeit und Unparteilichkeit verpflichtet ...; der Zugang zum Gericht ist nicht in unzumutbarer Weise erschwert; schließlich gewährleistet das Verfahrensrecht des Gerichts rechtliches Gehör und ein Mindestmaß an prozessualer Gleicheit der Beteiligten" 22 .

3. In Anbetracht solch begrenzter Kontrolle des sekundären Staatengemeinschaftsrechts vermöchte allein eine (Bundes-)Verfassungsbeschwerde direkt gegen das jeweilige Organisations-Vertragsgesetz einem Bediensteten dieses Verbands Abhilfe durch ein nationales deutsches Gericht in Aussicht zu stellen ${ }^{23}$. Doch wäre ein solcher außerordentlicher Rechtsbehelf in der Regel untauglich, da alsbald verfristet ${ }^{24}$. Zumindest dann nämlich, wenn in der Billigung des Gründungsvertrags bzw. einer späteren Beitrittsübereinkunft zugleich die vorweggenommene Zustimmung zu primärrechtsgemäßer Weiterentwicklung des Verbandsrechts beinhaltet ist, beginnt ein neuer Fristenlauf selbst dann nicht, wenn die Organisationsverfassung aufgrund in ihr niedergelegter Änderungsvorschriften gegenüber der ursprünglich akzeptierten Fassung umgestaltet wird ${ }^{25}$. Weil und soweit diese Modifikation zu ihrer international-rechtlichen wie innerstaatlichen Geltung keiner erneuten Einschaltung der gesetzgebenden Körperschaften bedarf, fehlte nämlich einer Anrufung des Bundesverfassungsgerichts der
Prüfungsgegenstand in Form eines konkreten landesrechtlichen Hoheitsaktes ${ }^{26}$.

Dies gilt aber auch für (bloß) abgeleitete Rechtssetzung innerhalb eines Verbands, ist deren zulässiger Rahmen doch durch den primärrechtlich niedergelegten Aufgaben- und Zuständigkeitsbereich des Hauptorgans abgesteckt $\mathrm{t}^{27}$. Allenfalls in dem wohl eher theoretischen Fall, daß das Organhandeln diese Grenzen (absichtlich) überschreitet ${ }^{28}$, könnte wieder die Frage nach innerstaatlichem Rechtsschutz hiergegen aktuell werden. Speziell im Dienstrecht aber wäre gerade dann vorab die verbandsinterne richterliche Kontrolle erfolgversprechend. Wenn nun dies gerichtliche Organ jedoch die Verbandsrechtmäßigkeit einer Maßnahme bestätigt, so wäre damit einem in der Angelegenheit noch angegangenen mitgliedstaatlichen Richter wohl verwehrt, anders zu befinden ${ }^{29}$. Ein Rechtsbehelf müßte demnach, wenn nicht schon wegen der fehlenden internationalen Zuständigkeit, doch zumindest deshalb scheitern, weil er in der Sache nicht begründet wäre ${ }^{30}$.

12 Vgl. bereits Gramlich, Europäische Zentralbank und Art. 24 Abs. 1 GG, 1979, 156 f.; ferner Rojahn, Art. 24, in: von Münch (Hrsg.), GG-Kommentar, Bd. 2, 2. Aufl. 1983, 99 (106 f. - Rdn. 18).

13 BVerfGE 59, 91; ähnlich - „Grundprinzip eines wirksamen Rechtsschutzes" - BVerfGE 58, 30 f., im Anschluß an Tomuschat, aaO, Rdn. 58 (47); in bezug auf fremdstaatliche Gerichtsentscheidungen ebenso BVerfGE 63, $375 \mathrm{ff}$.

${ }_{14}$ Noch immer ist dies nur ausnahmsweise der Fall; s. jedoch Art. 179 EWGV, 152 EAGV, und zu Art. 43 EGKSV Akehurst, The Law Governing Employment in International Organizations, 1967, 15 f.; Seyersted, ZaöRV 24 (1964) 1 (56 ff.).

15 So BVerfGE 59,94. S. außer den zuvor Genannten noch Seidl-Hohenveldern, aaO, 192f. (Rdn. 1351); Plantey, aaO, $425 \mathrm{f}$.; Brownlie, Principles of Public International Law, 3. Aufl. 1979, 687; Schreuer, Die Internationalen Organisationen, in: Neuhold/Hummer/Schreuer (Hrsg.), Österreichisches Handbuch des Völkerrechts 1, 1983, 154 (165).

${ }_{16}$ Hierzu näher Ballaloud, Le Tribunal Administratif de l'Organisation Internationale de Travail et sa Jurisprudence, 1967, 9 ff.; B. Knapp, in: Encyclopedia of Public International Law, Instalment 5 - International Organizations in General, 1983, $94 \mathrm{ff}$; zur Kontinuität ratione materiae in bezug auf das VölkerbundsTribunal s. a. Akehurst, aaO, 13 f.; Bastid, Rec. des Cours 92 (1957-II) 343 (368 ff.).

17 BVerfGE 59, 91

18 S. insbes. BVerfGE 60, 253 (303); 63, 332 (337f.); sowie bereits BVerfGE 59,280 ( $283 \mathrm{ff}$.).

19 Ähnlicher Ansatz schon bei Wengler, JZ 1965, 100 (101); kritisch dazu Frowein, Fs. BVerfGE, 1976, II, 187 (191 ff.).

20 Wie unlängst das Verwaltungsgericht der I.B.R.D. (s. Amerasinghe, I. C. L. Q. 31 [1982] 748 ff.; De Vuyst, R. B.D.I. 16 [1981/82] 81 ff.).

${ }_{21}$ Häufig werden die Gremien als „appeals board“ bzw. „commission de recours" bezeichnet, so etwa bei der O.E.C.D. (s. Hahn/Weber, Die OECD Organisation für Wirtschaftliche Zusammenarbeit und Entwicklung, 1976, 146), dem Europarat (Plantey, aaO, 425), der NATO (s. Vandersanden, La Commission de recours de l'O.T.A.N., R.B.D.I. 10 [1974] 90 ff.) und auch bei der E.S.A. (Staff Regulations, $33 \mathrm{ff}$. ).

22 BVerfGE 59, 91 f. Bei der E. S. A. etwa genügen die Regeln 33, 34, 36 und 41 des Personalstatuts, die vom Hauptorgan, dem Rat, aufgrund von Art. XI (5) lit. i) der Gründungskonvention vom 30.5.1975 erlassen wurden (s. BGBl. 1976 II, 1862), diesen Voraussetzungen voll und ganz.

${ }^{23}$ St. Rspr. seit BVerfGE 1, 396 (410); vgl. nur Kimminich, AöR 93 (1968) 485 (513); Bernhardt, Fs. BVerfG II, 154 (172 f.); Rojahn, Art. 59, in: von Münch, aaO, 789 (812ff. - Rdn. 44 f.).

24 Die Jahresfrist nach $\$ 93$ II BVerfGG läuft wie sonst auch ab Inkrafttreten des Zustimmungsgesetzes (s. aber (Maunz/) Schmidt-Bleibtreu (/Klein/Ulsamer), BVerfGG - Kommentar, 1964 ff., \$93 Rdn. 49, (24).

25 Hierzu Seidl-Hohenveldern, aaO, $231 \mathrm{ff}$. (Rdn. $1529 \mathrm{ff}$.), sowie Art. $312 \mathrm{ff}$. der Seerechtskonvention (Text bei: Platzöder/Graf Vitzthum, Seerecht, 1984, 69 ff.).

26 Angedeutet wird dies auch bei Bernhardt, BVerfG-Fs. II, 163.

27 Hierzu insbes. Ozieke, A. J. I. L. 77 (1983) 239 ff., sowie bereits Morgenstern, B. Y.I. L. 48 (1976/77) 241 (249 ff.).

28 Sofern etwa dem NATO- „Doppelbeschluß“ vom Dez. 1979 volle Rechtsverbindlichkeit für die Mitgliedstaaten beigemessen würde, obgleich solch imperative Handlungsform im Nordatlantikvertrag nicht vorgesehen ist (s. die Divergenzen zwischen Bleckmann, DVBI. 1984, 8 [10], von Münch, NJW 1984, 577 [581 mit N.60], Schweisfurth, ArchVR 22 [1984] 195 [196ff.], A. Weber, JZ 1984, 589 [592], und Däubler, Stationierung und Grundgesetz, 1982, 93 ff.), wäre ein hierauf gerichteter Wille der Teilnehmerstaaten ggf. als ultra vires-Handeln zu qualifizieren und daher jedenfalls kein rechtmäßiges Organhandeln mehr. Vom BVerfG wurde diese Frage freilich nur gestreift (s. das Urteil des 2. Senats vom 18.12.1984, NJW 1985, 603 [ff.]; ebenso bereits BVerfGE 58, 29; 59, 85.

${ }_{29}$ Zur Problematik allgemein Brownlie, aaO, 56; s. a. die Erwägungen in BVerfGE 37, 271 (281 f.), sowie Gramlich, G. Y.I.L. 27 (1984) i. E.

30 Dazu auch Meron, Rec. des Cours 167 (1980-II) 285 (356 und $381 \mathrm{f}$., N.205); ferner Toll $v$. Moreno, 458 U.S. 1 (1982). 
4. Allerdings läßt sich erwägen, ob nicht in derartigen Fällen eine anfechtbare Maßnahme deutscher öffentlicher Gewalt insoweit anzunehmen sei, als ein StaatenVertreter im Rahmen des Hauptgremiums der Internationalen Organisation ${ }^{31}$ durch sein (Abstimmungs-)Verhalten erst das Zustandekommen des Beschlusses dieses Organs ermöglicht ${ }^{32}$. Denn die umfassend formulierte, rechtsverbindliche Eingangsvorschrift des GG, wonach „(d)ie Würde des Menschen ... zu achten und zu schützen, ... Verpflichtung aller staatlichen Gewalt" sei, „(d)as Deutsche Volk sich darum zu unverletzlichen und unveräußerlichen Menschenrechten als Grundlage jeder menschlichen Gemeinschaft" bekenne - und dies nicht etwa nur im räumlichen Geltungsbereich des GG, sondern überall in der Welt ${ }^{33}$ ! -, schließlich die Grundrechte insonderheit „Gesetzgebung, vollziehende Gewalt und Rechtsprechung als unmittelbar geltendes Recht ... binden "34, läßt ja schwerlich einen anderen Schluß zu als den, daß auch hoheitliches Tätigwerden "nach außen“ nur nach Maßgabe verfassungs- und vorab grundrechtlicher Direktiven wie Schranken rechtens sei $^{35}$. Freilich kann hieraus allein keineswegs die totale Kongruenz der verfassungsrechtlichen Bindungen bei Handlungen mit auswärtigem Bezug mit denjenigen für Staatsakte innerhalb des deutschen Hoheitsgebiets postuliert werden. Identisch ist lediglich die Ausgangsposition, wie sie an der konstitutionell (und konstitutiv) begrenzten Hoheitsmacht im demokratischen Verfassungsstaat anknüpfen muß ${ }^{36}$. Das Grundgesetz enthält nämlich gerade für die grenzüberschreitend wirkenden Aktivitäten deutscher Staatsorgane auch Verhaltensmaßregeln hinsichtlich deren konkreter Gestaltung, genauer: es statuiert gewisse definitive (Selbst-)Beschränkungen staatlichen Tuns und Lassens ${ }^{37}$. Gemeinhin in einer Zusammenschau als Aussage(n) über eine „völkerrechtsfreundliche Haltung “ der Verfassung interpretiert ${ }^{38}$, verbieten diese Normen nicht bloß programmatisch, sondern mit unmittelbarer Rechtsverbindlichkeit ${ }^{39}$ die Störung des friedlichen Zusammenlebens der Völker, insbesondere (vorbereitende) Maßnahmen zur Führung eines Angriffskriegs ${ }^{40}$, lassen jedwede Vereinigungen nur $\mathrm{zu}$, wenn sie nicht gegen den Gedanken der Völkerverständigung gerichtet sind $^{41}$, bekunden den Willen des Staatsvolks - und verpflichten mithin dessen besondere Organe ${ }^{42}-$, dem Frieden der Welt zu dienen ${ }^{43}$, befürworten die Mitwirkung an der Herbeiführung und Sicherung einer friedlichen und dauerhaften Ordnung zwischen den Völkern der Welt ${ }^{44}$ und sehen zur Regelung zwischenstaatlicher Streitigkeiten den Abschluß von Vereinbarungen über eine universelle und obligatorische Schiedsgerichtsbarkeit vor $^{45}$. Der hierbei aus verschiedenen Blickwinkeln umschriebene Friedensauftrag des GG mag durchaus den Staatszielbestimmungen an die Seite gestellt werden, wie sie herkömmlicherweise allein durch Art. 20 I GG fixiert sein sollen ${ }^{46}$; wegweisend begrenzt er die Handlungsmöglichkeiten der Träger deutscher hoheitlicher Gewalt (über das völkerrechtlich Gebotene hinaus), wirkt sich als rechtsverbindliches Leitprinzip auf alle Verfassungsbereiche aus, die für den Völkerfrieden von Belang sind.

Mit solch positiver Inhaltsbestimmung ${ }^{47}$ von verfassungsrechtlicher Völkerrechtsoffenheit sind aber nicht immer oder gar notwendig auch erweiterte Kompetenzen zur Einschränkung grundrechtlich verbürgter Freiheitspositionen verknüpft ${ }^{48}$. Wohl aber dürfen die auswärtsgerichteten Maßgaben bei der normativen Ausfüllung der jeweiligen Gesetzes- bzw. Regelungsvorbehalte berücksichtigt werden, was insoweit nicht zuletzt im Wege vertragsgesetzlicher Billigung bestimmter Organisationsstatuten erfolgen wird ${ }^{49}$. Kaum sachlich gerechtfertigt erscheint es jedoch, die nationale (Ein-/Rück-) Bindung bereits dann für verfassungsrechtlich ausreichend zu erachten, wenn sie lediglich punktuell und auf einzelne Akte der Legislativorgane beschränkt ins Werk

31 Vgl. Seidl-Hohenveldern, aaO, 136 ff. (Rdn. 1101 ff.); Hahn, Funktionenteilung im Verfassungsrecht europäischer Organisationen, 1977, $62 \mathrm{f}$.

$32 \mathrm{Zu}$ solcher Aufspaltung s. bereits Scelle, Fs. Wehberg, 1956, 324 (336 und $341 \mathrm{f}$.$) ; ferner Meng, Das Recht der Internationalen Organisationen - eine Ent-$ wicklungsstufe des Völkerrechts, 1979, 47; Schreuer, ArchVR 22 (1984) 363 (390 f.). Aufschlußreich in diesem Kontext auch Steindorff, RIW/AWD 1984, 767 (769f.) im Hinblick auf Richtlinien nach Art. 100 EWGV, sowie der „Interim Award Regarding Jurisdiction“ des ICC-Schiedsgerichtshofs vom 5.3.1984 in Sachen Westland Helicopters Ltd. v/ Arab Organization for Industrialization et al., I. L. M. 23 (1984) 1071 (1085 f. - III. 2. E.)

${ }^{33}$ Zur Auslegung dieses Merkmals insbes. von Münch, Art. 1, in: ders. (Hrsg.), GG-Kommentar, Bd. 1, 3. Aufl. 1985, 65 (100 f. - Rdn. $41 \mathrm{ff}$.).

${ }^{34}$ Hierzu eingehend M.Schröder, Fs. Schlochauer, 1981, 137 (138ff.); von Münch, aaO, 97 (Rdn. 49); Bernhardtl, BVerfG-Fs. II, $178 \mathrm{f}$.

35 Entscheidend hierfür ist nicht der Bezug zum räumlichen Geltungsbereich des GG (Art. 23), sondern die umfassende Bindung der Staatsorgane gemäß Art. 20 III GG. Mißverständlich daher von Münch, aaO, 83 (Rdn. 30), wo allein der Territorialitätsaspekt angesprochen wird; zutreffend hingegen Kunig, Anm. zum Urteil des OVG Münster vom 26.11.1981, DVBl. 1983, 37 (38f.), sowie Podlech, Art. 1 Abs. 1, im Alternativ-Kommentar zum GG, Bd. 1, 1984, 273 (298) (Rdn. 81), und Denninger, Art. 1 Abs. 2, 3, ebd., 298 (303 - Rdn. 6 - und 307 - Rdn. 15).

36 Ebenso M. Schröder, Fs. Schlochauer, 138; Bleckmann/Busse, DVBl. 1977 794 (796); Bernhardt, BVerfG-Fs. II, $178 \mathrm{f}$

37 Vgl. etwa G. Frank, im Alternativ-Kommentar, Bd. 2, 1984, F. I./Rdn. $34 \mathrm{ff}$. (1511 ff.); kritisch allerdings Geck, VVDStRL 36 (1978) 142 (144).

38 So bereits Mosler, Das Völkerrecht in der Praxis der deutschen Gerichte, 1957, 9 f.; Bleckmann, DöV 1979, 309 ff.; Zuleeg, im Alternativ-Kommentar, Bd. 1, C., Art. 24 Abs. 3, Art. $25 /$ Rdn. 60 ff. (202 f.); s. a. BVerfGE 18, 112 (121); 58,41 .

39 S. bereits Nawiasky/Leusser, Die Verfassung des Freistaates Bayern Handkommentar, 1948, 78, zum Vorspruch einer Landesverfassung, die ebenfalls den "festen Entschluß“ des Bayerischen Volkes bekundet, „den kommenden deutschen Geschlechtern die Segnungen des Friedens, der Menschlichkeit und des Rechtes dauernd zu sichern “; ferner Hernekamp, Art. 26, in: von Münch, aaO, II, 167 (167 - Rdn. 1); Frank, aaO, F. I./Rdn. 30 f. (1511).

${ }^{40}$ Art. $26 \mathrm{I} 1 \mathrm{GG}$ knüpft an Art. 1 (2), 2 (4) der U.N.-Charta an (so Menzel, Art. 26, im Bonner Kommentar, II, 1., 4.c [6, 12]; Hernekamp, aaO, Art. 26 Rdn. 3 [168], 8 [169]; Frank, aaO, F. I./Rdn. 39 ff. [1513 ff.]).

41 Der Normbereich des Art. 9 II reicht über den des Art. 26 GG hinaus (Scholz, Art. 9 [1979], in: Maunz/Dürig, GG-Kommentar, 6. Aufl. 1982 ff., Rdn. 131 [101]; anders wohl Ridder, im Alternativ-Kommentar 2, E., Art. 9 Abs. $2 /$ Rdn. $38 \mathrm{ff}$. [1465]).

42 Von Münch, Präambel, in: ders., aaO, 1, 1 (6 - Rdn. 12); Zuleeg, im Alternativ-Kommentar 2, B./Rdn. 19 f. (139); ähnlich bereits Abendroth, EurArch 1951, 4385 (4391). Die Darlegungen im Urteil des AG Schwäbisch Gmünd vom 16.10.1984 (NJW 1985, 211 [212]) werden dieser Bindung schwerlich gerecht.

${ }^{43}$ So die zweite Präambel-Erwägung, und ebenso der vorerwähnte bayer. Verfassungsvorspruch. Bemerkenswerterweise findet dies in der Kommentierung von Maunz (1976) - in Maunz/Dürig, aaO - überhaupt keine Erwähnung und kommt auch bei Schweisfurth, Fs. Mosler, 1983, $857 \mathrm{ff}$., nur am Rande vor (ebd. 863), ebenso bei Zuleeg, aaO, B./Rdn. 107 (163), wo immerhin noch auf die Konkretisierung durch Art. 26 hingewiesen wird. Vgl. demgegenüber aber von Mangoldt/Klein, Das Bonner Grundgesetz, Bd. 1, 2. Aufl. 1957, 44, 45.

44 Art. 24 II GG, wo gleichfalls eine Beziehung zu Art. 1 und 2 der U. N.-Charta herstellbar ist (s. Frank, aaO, F. II., Art. 24 Abs. 2/Rdn. 5 [1533 f.], 8 [1535 f.]; Rojahn, aaO, Art. 24/Rdn. 52 [129]; wohl auch Maunz, Art. 24 [1964], in: Maunz/ Dürig, aaO, Rdn. 25 [22]).

45 Dem Gebot grammatikalischer Interpretation dürfte bei Art. 24 III GG noch Genüge getan sein, wenn ganz allgemein international-rechtliche Streitigkeiten zwischen Völkerrechtssubjekten unter diese Vorschrift eingeordnet werden (so Maunz, aaO, Art. 24/Rdn. 31 [26]; Füsslein, Art. 24, in: Seifert/Hömig (Hrsg.), Grundgesetz - Taschenkommentar, 1982 Rdn. 6 [192 f.]).

46 So insbes. Frank, aaO, F. I./Rdn. $30 \mathrm{ff}$. (1511 ff.), im Anschluß an Überlegungen Scheuners (Fs. Forsthoff, 1974, 325 [336f.]).

${ }_{47}$ Zum „positiven“ Friedensbegriff s. Frank, aaO, F. I./Rdn. 9 ff. (1504ff.), $28 \mathrm{f}$. (1510f.); ferner Murswiek, Der Staat 23 (1984) 523 (535 f.).

48 Schröder, Fs. Schlochauer, $140 \mathrm{f}$.; ders., in: Haungs (Hrsg.), Verfassung und politisches System, 1984, 67 (72); anders wohl Doehring, VVDStRL 32 (1974) 7 (39 ff.); differenzierend Isensee, ebd., 49 ( $57 \mathrm{ff}$.); Herzog, ebd., $122 \mathrm{f}$.

49 So grundsätzlich auch Rojahn, aaO, Art. 24/Rdn. 31 (117f.), der aber hernach (Rdn. 36-120) die oben dargelegte (dritte) Möglichkeit nicht erörtert. Tomuschat (aaO, Art. 24 Abs. $1 /$ Rdn. 49 [41], 62 [50]) bekundet zwar überzeugend, „der Vertrag über die Gründung einer zwischenstaatlichen Einrichtung (dürfe) selbst von den Grundrechtsgewährleistungen des GG in einem Maße abweichen..., welches von den normalen Vorbehaltsschranken nicht mehr gedeckt wäre. Soweit ersichtlich, ist aber derartiges bisher niemals geschehen" (ebd., Rdn. 64 [50]), begreift freilich andererseits meine früheren Darlegungen (aaO, $1979,148 \mathrm{f}$.) schwerlich zutreffend als in der Tat abzulehnende "Hypotheken“Theorie... Im übrigen sieht das GG ansonsten selbst für den Notstand nur einzelne Grundrechtsverkürzungen vor (Füsslein, Art. 115 c, in: Seifert/Hömig, aaO, Rdn. 3 [537]). 
gesetzt wurde ${ }^{50}$. Vielmehr sollte auch hier ein vergleichender Blick auf den innerstaatlichen Gesetzesvollzug und die hiermit befaßten Rechtsanwender verdeutlichen, daß ein Gebrauchmachen von verfassungsgemäß zugeordneten Kompetenzen stets nur in den Bahnen höherrangiger Normen vonstatten gehen darf ${ }^{51}-$ alle vollziehende Gewalt erscheint sohin an Gesetz und Recht gebunden ${ }^{52}$, ohne daß es maßgeblich auf den territorialen Bezug des Verhaltens bzw. von dessen Auswirkungen zum grundgesetzlichen Geltungsbereich ankäme. Allenfalls mag (zuweilen) die übergesetzliche Verbindlichkeit einer allgemeinen Völkerrechtsregel nach Art.25 GG denjenigen Hoheitsakten, die hiernach unzulässig im Ausland gesetzt wurden, bereits die innerstaatliche Geltung versagen ${ }^{53}$. Nun besteht jedoch gerade keine Norm des Völkergewohnheitsrechts, welche die Handlungen (fremd)staatlicher Organwalter in ihrer Eigenschaft als Mitglieder von Gremien intergouvernementaler Verbände den generellen Regeln über den einzelstaatlichen (räumlichen) Jurisdiktionsbereich unterwerfen würde ${ }^{54}$. Sie sind mithin nicht etwa allein deshalb schon unzulässig, weil sie sich regelmäßig - aus der Warte fast aller Organisationsbeteiligten - außerhalb von deren Territorien vollziehen. Der jeweilige Sitzstaat eines Verbands gestattet $^{55}$ vielmehr mit der (völkervertraglich wirksamen) Erlaubnis für die Tätigkeitsaufnahme einer internationalen Einrichtung und bis zu deren Wiederaufhebung organschaftlich ausgeübte Verbandsfunktionen ganz generell, jedenfalls soweit dabei kein offenkundiges ultra vires-Verhalten in Frage steht. Da nun aber im aus staatlichen Amtswaltern zusammengesetzten Hauptorgan Willensbildung und Beschlußfassung notwendig ambivalente Züge aufweisen, sowohl Verbands- als Staaten-Handeln sind, erstreckt sich die Zustimmung des Sitzstaates objektiv auch auf beide Verhaltensaspekte. Das Verbot extraterritorialer Hoheitsausübung erfährt insofern gleichsam eine Bereichsausnahme ${ }^{56}$. Diese umfaßt wohl auch die Aktionen der im Verbandsorgan vereinigten Vertreter der Mitgliedstaaten, welche sich nicht (mehr) als Maßnahmen im Rahmen der Organisationsverfassung begreifen lassen - weder sub- noch objektiv - , sondern eigenständige Vertragsqualität aufweisen $^{57}$. Derart „uneigentliche" Verbandsbeschlüsse bedürfen freilich eines speziellen innerstaatlichen Geltungs- (und Anwendungs-)Befehls, vermögen also nicht unmittelbare normative Wirkungen in den Mitgliedstaaten zu zeitigen ${ }^{58}$, noch erlangen sie Verbindlichkeit innerhalb der staatengemeinschaftlichen Rechtsordnung ohne eine besondere Inkorporation und nur nach Maßgabe dieser Einbeziehung ${ }^{59}$.

5. Bei Geltung einer Einstimmigkeitsregel im Rat, wie dies noch immer in der Mehrzahl der zwischenstaatlichen Verbände klassischen Typs niedergelegt ist ${ }^{60}$ oder doch praktiziert wird ${ }^{61}$, wäre es dem deutschen Staatenvertreter im Verbandshauptorgan von Völkerrechts wegen sohin keineswegs versagt, das nach dem Grundgesetz „Verfassungsnähere “62, wenn nicht gar Gebotene ${ }^{63}$ auch durchzusetzen: Seine Veto-Position ließe sich effektiv dahingehend konkretisieren, daß eine aus deutscher, grundgesetzlicher Sicht bedenkliche Beschlußfassung gar nicht erst erfolgt(e). Das Bundesverfassungsgericht hat zwar bereits im ersten Verfahren, in welchem es zu außenpolitischen Streitpunkten Stellung zu nehmen aufgerufen war, berechtigte Zweifel geäußert, ob eine (einstweilige) „Anordnung, die dem verantwortlichen Leiter der Außenpolitik der Bundesrepublik die Abgabe einer
Erklärung von bestimmten, rechtlich erheblichem Inhalt ... zur Pflicht machen würde, überhaupt im Rahmen der Verfassungsgerichtsbarkeit läge und nicht vielmehr als unzulässige Einengung des dem Bundeskanzler hier einzuräumenden politischen Ermessens erachtet werden müßte" ${ }^{64}$. Die Bedenken, den zuständigen Organen ein positives Tun vorzuschreiben, greifen freilich gegenüber bloßen (zeitlich begrenzten) Verboten nicht im selben Maße durch; zumindest im Hinblick auf die Ratifikation völkerrechtlicher Verträge sah auch das Karlsruher Gericht eine Anweisung an die Bundesregierung, einen Notenaustausch einstweilen zu unterlassen, offenbar wenngleich nicht explizit - für keine Überschreitung seiner judiziellen Kompetenzen $\mathrm{an}^{65}$. Gerade wegen der Unanimität jedoch könnte eine Verpflichtung der (Organe der) Bundesrepublik Deutschland, in einer bestimmten oder zumindest nicht in einer bestimmten anderen Weise zu votieren, nur vor dem Zeitpunkt der endgültigen Ratsentscheidung (verwaltungs-)gerichtlich angestrebt werden. Hernach hätte sich ein hierauf zielender Antrag erledigt ${ }^{66}$, denn der einmal gefaßte Verbandsbeschluß bindet in aller Regel sämtliche Mitgliedstaaten, er ist für und gegen sie wirksam, weil seine verpflichtende Kraft bereits durch die in der Mitgliedschaft manifestierte Zustimmung anerkannt wurde. Den Verbandsparteien ist überdies aufgegeben, sich beschlußkonform zu verhalten, gegenbenenfalls auch innerstaatliche Durchführungsmaßnahmen zu ergreifen ${ }^{67}$. (Ungeschriebenes $)^{68}$ Organisationsverfahrensrecht beläßt immerhin

50 Angeschnitten wird dies Problem auch bei Rojahn, aaO, Art. 24/Rdn. 29 (117). Zur Rechtslage in den E. G. s. Bieber, in: Beutler/Bieber/Pipkorn/Streil, Die Europäische Gemeinschaft, 2. A. 1982, 98 (120 f.).

51 Åhnlich Herzog, Art. 20 (1980), in: Maunz/Dürig, aaO, VI./Rdn. 96 (250), VII./ Rdn. 82 (293).

s2 Vgl. Herzog, ebd., VI./Rdn. 32 (220), 37 (221 f.).

53 Vgl. Rojahn, aaO, Art. 25 / Rdn. 39 (164); BVerfGE 23, 288 (300); Geck, Fs. BVerfG II, 1976, 125 (138f.).

54 Positive Völkerrechtsregeln über die extraterritoriale Geltung staatlicher Normen existieren wohl überhaupt nicht (s. Rudolf, BerGesVR 11 (1973) 7 (17); Rojahn, aaO, Art. $25 /$ Rdn. 22 (150); Gramlich, Rechtsgestalt, Regelungstypen und Rechtsschutz bei grenzüberschreitenden Investitionen, 1984, 374 ff.).

55 Beispiele für derartige Abmachungen bei Hailbronner, G. Y.I. L. 22 (1979) $313 \mathrm{ff}$; s. jüngst auch Valleix, R. G. D.I.P. 88 (1984) 621 (642 ff.).

56 Insoweit durchaus ähnlich der völkerrechtlichen Zulässigkeit von nachrichtendienstlichen Auslandsaktivitäten (dazu Rieger, ZRP 1985, 3 [7]; Gusy, DV 1984,273 [283]).

57 S. etwa Seidl-Hohenveldern, aaO, 1984, Rdn. 1115 (141); (Beutler/)Bieber (I Pipkom/Streil), aaO, 128.

58 Eine Bindung lediglich der Mitgliedstaaten selbst dürfte aber wohl nicht die Regel sein (s. H. P. Ipsen, Europäisches Gemeinschaftsrecht, 1972, 341 f.).

59 Seidl-Hohenveldern, Fs. Mosler, 1983, 881 (886).

60 Beispiele bei Schermers, International Institutional Law, II, 1972, $326 \mathrm{ff}$; ferner etwa Art. 30, 32, 43 des Gründungsvertrags der Lateinamerikanischen Integrationsvereinigung (ALADI) vom 12.8.1980 (I. L. M. 20 [1981] 672 ff.), wo eine abgeschwächte Form - keine Gegenstimmen bei etlichen bedeutsamen Ratsbeschlüssen - vorgesehen ist; Art. 5 (1) (a), 6 (1), (5) des Vertrags über die Errichtung der Organisation ostkaribischer Staaten (OECS) vom 18.6.1981 (I.L. M. 20 [1981] $1166 \mathrm{ff}$.), die offenbar den Vorschriften der OECD-Konvention (Art.6 [2], [3]) nachempfunden sind; Art. 7 (VII) des Protokolls vom 12.2.1981 zur Änderung des Internationalen Übereinkommens vom 13.12. 1960 über Zusammenarbeit und Sicherung der Luftfahrt „Eurocontrol“... (BGBl. 1984 II, 71).

61 Beispielsweise in Verfolg der Luxembourger Beschlüsse vom 28./29.1.1966 im Hinblick auf den Rat der E.G. (hierzu Schweitzer, Fs. Armbruster, 1976, 75 [80 ff.]; Everling, Gs. Constantinesco, 1983, 133 [137]; Seidl-Hohenveldern, ebd., $695[697 \mathrm{ff}]$.

${ }_{62}$ S. etwa BVerfGE 4, 157 (168); 12, 281 (290ff.); 62, 169 (186).

63 Eine Beschränkung der Handlungsmöglichkeiten auf eine einzige mag gerade im außenpolitischen Bereich nur höchst selten vorkommen (s. BVerfGE 55, 349 [365, 368]; aber auch Ress, in: Rechtsfragen grenzüberschreitender Umweltbelastungen, 1984, $85[99$, N. 43]).

64 BVerfGE 1, 281 (282); zustimmend Bernhardt, BVerfG-Fs. II, $175 \mathrm{f.}$

65 BVerfGE 35, 193 (196); 35, 257 (262); 38, 49 (51); s. a. R. Hahn, Pub. L. 1984,530 (535), zu einem ähnlichen Fall in Kanada ([1984] 3 D. L. R. [4 $\left.{ }^{\text {th }}\right] 193$ ).

${ }_{66} \mathrm{Da}$ dem Kläger mit einer Entscheidung über das ursprüngliche Begehren nicht mehr gedient wäre, dürfte eine analoge Anwendung des $\$ 11314$ VwGO in Betracht zu ziehen sein (s. Kopp, VwGO, 6. A. 1984, S113/Rdn. 107 [998]).

67 Meng, aaO, $1979,76$.

68 Letzlich beruhend auf der "clausula rebus sic stantibus" (s. Art. 62 der Wiener Vertragsrechtskonventioin "1969); s. etwa die Regelung in Art. 2 der Geschäftsordnung des Rats der E. G. (ABl. EG 1979, L 268, 1). 
jedem Mitgliedstaat die Befugnis, denselben Verhandlungsgegenstand bei (vorgeblich) veränderter Sachlage oder doch aus ähnlichem Anlaß zur erneuten Beratung und Beschlußfassung vorzuschlagen ${ }^{69}$. Dabei ist nunmehr zunächst lediglich eine individuelle Novellierungsinitiative vorhanden, deren Erörterung oder gar Verwirklichung auch vom jeweils aktiv gewordenen Mitgliedsland nicht erzwungen werden kann. Dem betroffenen Individuum bliebe dann allenfalls die Möglichkeit eines Fortsetzungsfeststellungsbegehrens, als Voraussetzung der Geltendmachung von Staatshaftungsansprüchen gegen die Bundesrepublik Deutschland ${ }^{70}$. Hierbei müßte er allerdings gleichfalls gewärtigen, daß gemäß $\ 5 \mathrm{Nr} .2$ $\mathrm{RBHG}^{\mathrm{1}}$ eine amtliche Erklärung des Bundeskanzlers dem tätig gewordenen Amtswalter bescheinigen würde, sich entsprechend politischen oder internationalen Rücksichten verhalten zu haben. Wiewohl nun die Verfassungskonformität dieser Vorschrift in der Literatur häufig in Zweifel gezogen wurde ${ }^{72}$, so steht doch nach dem Erkenntnis des Bundesverfassungsgerichts zum Gegenseitigkeitserfordernis bei der Amtshaftung gegenüber Ausländern $^{73} \mathrm{zu}$ erwarten, daß diese Ausnahme vom Grundsatz des Art. 34 GG ebenfalls als noch verfassungsgemäß erachtet werden wird und damit auch dieser Weg für den Geschädigten versperrt bleibt ${ }^{74}$.

6. Vorbeugenden Rechtsschutz auch im hier bezeichneten Kontext prinzipiell für statthaft zu erachten, sollte zumindest dann erwogen werden, wenn ein mehrphasiger Verfahrensgang bis zur abschließenden Beschlußfassung im Verbandshauptorgan vorgesehen ist. Ein wohl nicht völlig außergewöhnliches Beispiel für solche Komplexität stellt die Gehaltsanpassungsregelung im Rahmen der „Koordinierten Organisationen“ dar. Hier vermag das in der Judikatur geforderte, qualifizierte, gerade auf die Inanspruchnahme präventiven gerichtlichen Schutzes gerichtete Interesse ${ }^{75}$ sich an den Umstand zu knüpfen, daß der sich selbst allenfalls hortatorischen Charakter beimessende ${ }^{76}$ „Bericht “ des nicht förmlich institutionalisierten „Co-ordinating Committee of Government Budget Experts" - er wird regelmäßig zur Gehaltsstruktur aller in den angeschlossenen Verbänden Beschäftigten erarbeitet und äußert sich insgesamt zu dieser wie auch zu den je vorgesehenen Anpassungsverfahren ${ }^{77}$ - in der Praxis weithin vollendete Tatsachen schafft. Die von diesem Gremium ausgesprochenen „Empfehlungen“" werden von allen beteiligten zwischenstaatlichen Einrichtungen akzeptiert, ohne daß über ihren Inhalt in den Verbandsorganen noch eingehender debattiert oder gar Änderungen beschlossen würden ${ }^{78}$. Die de facto-Automatik nötigt dazu - zumal der ansonsten zu gewärtigende Einwand der Gesetzesumgehung bei derartigen Konstellationen nichts verschlägt, weil ein Vorverfahren bei Akten oberster Staatsorgane generell nicht stattfin$\operatorname{det}^{79}$-, ettektiven Rechtsschutz nach dem Verfassungsgebot des Art. 19 IV GG durch Zulassung außergewöhnlicher Rechtsbehelfsmöglichkeiten zu gewährleisten ${ }^{80}$. Es geht insonderheit bei den skizzierten Dienstrechtsangelegenheiten eben nicht (bloß) um "Regierungsakte" rein staatspolitischer Natur ${ }^{81}$, die sich allein in einem Raum notwendig breitgefächerter Gestaltungsfreiheit ins Werk setzen ließen und die wegen fehlender direkter Grundrechtsberührung keiner gesteigerten Legitimation bedürften. Vielmehr ist hier internationales Verwaltungs-, nämlich Bedienstetenrecht nicht etwa erst „im Werden" begriffen ${ }^{82}$, sondern partiell bereits höchst detailliert in Geltung. Seine völkerrechtliche Abkunft wie sein gleichartiger Geltungsgrund reichen nicht hin, um die in der (Teil-)Identität der Regelungsadressaten begründete materielle Ähnlichkeit mit nationalen öffentlichen Dienstrecht zu negieren. Das Bundesverfassungsgericht hob hierzu im zweiten „Eurocontrol“-Beschluß zutreffend hervor, zwar könne dahinstehen, „ob und inwieweit die innerstaatlichem Recht entnommenen Kriterien für die Abgrenzung zwischen öffentlichem und privatem Recht auf das interne Personalrecht einer internationalen Organisation überhaupt übertragbar seien "83; die einschlägigen staatengemeinschaftlichen Bestimmungen gingen jedenfalls zweifelsohne von Vorstellungen des Landesrechts aus und ähnelten in mehrfacher Hinsicht gerade dem mitgliedstaatlichen Beamtenrecht ${ }^{84}$. Dem läßt sich nurmehr hinzufügen, daß selten eine einzige, etwa die französische Landesrechtsordnung den international-rechtlichen Normierungen zugrunde liegt, sondern immer häufiger die Regelungsmuster mehrerer Verbandsmitgliedsländer als Basis harmonisierender „internationaler Gesetzgebung "85 dienen ${ }^{86}$.

7. Nun läßt sich freilich kaum verkennen, daß ein jegliches Verbandsorgan, da es seine Existenz allein dem Organisationsgründungsakt schuldet, nur im Rahmen der eigenen, autonomen Rechtsordnung tätig werden darf und $\mathrm{kann}^{87}$. Es unterliegt dabei zum einen dem Primär-, dem Verfassungsrecht der Einrichtung, hat aber darüber hinaus auch die selbsterzeugten Normen zu achten, solange und soweit sie nicht im hierfür vorgesehenen Verfahren geändert oder gar völlig aufgehoben

69 Einzelheiten bei Seidl-Hohenveldern, aaO, 1984, 143 ff. (Rdn. $1121 \mathrm{ff}$.). Daß eine verfassungsrechtliche Verpflichtung hierzu besteht, hebt Frowein hervor (BVerfG-Fs. II, 205).

70 Zum diesbezüglichen Feststellungsinteresse s. Kopp, aaO, $\ 113 / \mathrm{Rdn} .58$ (971 f.), 108 (999).

71 Gesetz über die Haftung des Reichs für seine Beamten vom 22.5.1910 (BGBl. III 2030-9).

72 S. nur Weiss, Auswärtige Gewalt und Gewaltenteilung, 1971, 83 f.; Dagtoglou, Art. 34 (Zweitbearb. 1970), im Bonner Kommentar, Rdn. 323 ff. (139 ff.); Gramlich, BayVBl. 1983, 485 (488).

${ }_{73}$ NVwZ 1983, 89; auszugsweise auch in MDR 1983, 107; dazu Gramlich, BayVBl. 1983, $485 \mathrm{ff}$.

74 Für verfassungsrechtlich unbedenklich halten die Ausschlußnorm etwa W. Meyer, Art. 34, in: von Münch, aaO, II, 393 (413 - Rdn. 64); Rittstieg, Art. 34, im Alternativ-Kommentar, aaO, II, Rdn. 23 (221).

75 S. unlängst Peine, Jura 1983, 285 ff.; ferner etwa BVerwG, DVBI. 1971, 746 (747), DöV 1983, 980 (981).

${ }^{76} \mathrm{Zu}$ intergouvernementalen Beschlüssen von „unbestimmter rechtlicher Beschaffenheit ${ }^{*}$ eingehender Hahn, aaO, 1977, $74 \mathrm{ff}$.

77 Näheres zum 191. Bericht (vom 16.2.1983 - „Revision of the Remuneration Adjustment Procedure for Staff of the Coordinated Organisations and Triennial Review of Salaries at 1 $1^{\text {st }}$ July, 1982“ (CCG [83] 1)) bei Gramlich, ZBR 1985 , 38 .

78 So Plantey, aaO, 52; zu Art und Umfang dieser Besoldungskoordination s. ebd., $50 \mathrm{ff}$. Vor kurzem geriet das sonst eher reibungslose Übernahme-Verfahren allerdings ins Stocken, und beteiligte Verbände wichen (auf der Basis dies gestattender eigener Regelungen) von den Empfehlungen ab.

79 S 126 III Nr. 1 BRRG als Ausnahme von SS 68 I 2 Nr. 1, II VwGO bezieht sich allein auf Beamte der in S121 BRRG angeführten, deutschen Dienstherrn.

${ }_{80}$ In wenngleich anderem Zusammenhang betont von Umbach, DVBI. 1971, 741 (742f.), in einer Anm. zu BVerfGE 31, $364 \mathrm{ff}$.

81 Vgl. dazu Schenke, Art. 19 Abs. 4 (Zweitbearb. 1982), im Bonner Kommentar, Rdn. 219 ff. (121 ff.).

82 So der Titel eines von Schwarze hrsg. Sammelwerks: „Europäisches Verwaltungsrecht...“, 1982; s. a. Everling, DVBl. 1983, 649 ff.; Rengeling, EuR 19 (1984) $331 \mathrm{ff}$.

83 BVerfGE 59, 88.

84 S. a. Bleckmann, Internationale Beamtenstreitigkeiten vor nationalen Gerichten, 1981, 72 ff. und 107, sowie (in der Bewertung abweichend) Seidl-Hohenveldern, Die Immunität internationaler Organisationen in Dienstrechtsstreitfällen, $1981,34,58 \mathrm{ff}$. und $65 \mathrm{ff}$.; ders., Fs. Schlochauer, 1981, 615 (622 f.); vgl. ferner schon Guggenheim, Fs. Wehberg, 1956, 133 (143 f.); Zemanek, ZaöRV 24 (1964) 453 (467f.); Meng, aaO, 1979, $82 \mathrm{f}$.

85 Hierzu Skubiszewski, International Legislation, in: Encyclopedia of Public International Law 5, 97 (101).

${ }_{86}$ Seidl-Hohenveldern, aaO, 1984, 132 (Rdn. 1042); Downes, L. Qu. Rev. 101 (1985) 98 (112f.).

87 Dabei stehen sich freilich, wie Everling zutreffend hervorhebt (Gs. Constantinesco, $147 \mathrm{f}$.), Staatengemeinschaftsrecht einerseits und Völker- wie auch nationales Recht zum anderen nicht etwa unverbunden und undurchdringlich gegenüber. 
wurden $^{88}$. Auch die Räte Internationaler Organisationen sind mithin auf die Erfüllung je spezifisch gemeinsamer und gemeinschaftlicher Aufgaben verpflichtet ${ }^{89}$. Gerade im Dienstrecht dieser Institutionen steht dabei das Gebot der Gleichbehandlung der Beschäftigten ${ }^{90}$, das Verbot einer Differenzierung nach deren nationaler Herkunft und Zugehörigkeit ${ }^{91}$ im Mittelpunkt einer jeden Personalordnung. Nun ist es jedoch keine völlig außergewöhnliche Erscheinung, daß Amtswalter gleichzeitig den Vorschriften mehrerer Rechtsordnungen Folge zu leisten haben; wenn dies also auch für die einzelnen Staatenvertreter in internationalen Organen der Fall wäre - deren Bindung an (primäres) Verbandsrecht dürfte allerdings schwerlich unmittelbarer Art sein, sondern eher über das Zusammenwirken im Verbandsgremium vermittelt ${ }^{92}$, ihre Verpflichtungen bestehen lediglich diesem gegenüber -, so zeigte dies nur Strukturähnlichkeiten föderativen Ursprungs auf. Beispielsweise unterliegen in den Gliedstaaten eines Bundesstaates Behörden und Gerichte zuweilen sowohl zentralen als auch landesgesetzlichen Anordnungen, im Falle der Bundesrepublik sohin etwa bundesrechtlichen Vorschriften und den - nicht aufgehobenen oder doch unanwendbar gewordenen ${ }^{93}$ Bestimmungen der Länderverfassungen ${ }^{94}$. Anerkanntermaßen haben hierbei die Länder-Organe bei ihrem hoheitlichen Vorgehen nach Möglichkeit - d.h. im Rahmen der vom Bundesrecht eingeräumten Gestaltungs- oder Ermessenräume - ihr eigenes Verfassungsrecht $\mathrm{zu}$ befolgen, müssen also nicht zuletzt dessen Grundrechtsgewährleistungen beachten ${ }^{95}$.

Um nichts anderes geht es aber der Sache nach beim Wirken deutscher Staatsrepräsentanten in zwischenstaatlichen Einrichtungen. Dabei bleibt auch zu beachten, daß die so gewahrte Rückbindung an nationales (Verfassungs-)Recht zugleich im objektiven Interesse jedes internationalen Verbands liegen dürfte, weil sich diese Beziehung doch zuvörderst als ein Instrument zur Erhaltung der Funktionsfähigkeit der Organisation begreifen läß $\mathrm{t}^{96}$.

8. Gegenüber der aufgezeigten Möglichkeit, vor nationalen deutschen Gerichten (vorbeugend) Rechtsschutz zu erlangen, verschlägt es nichts, daß diplomatischer Schutz gegen Akte intergouvernementaler Organe nur soweit ausgeübt werden kann, wie dies das jeweilige Verbands-Primärrecht zuläßt; andere an sich nach allgemeinem Völkerrecht gegebene Protektionsmittel stehen dagegen kraft Organisationsmitgliedschaft und für deren Dauer nicht zu Gebote ${ }^{97}$. Auch in diesem Punkt erweist sich also ein weiteres Mal die wechselseitige Anreicherung von staatengemeinschaftlichem und zwischengliedstaatlichem Recht ${ }^{98}$. Denn attackiert würde im innerstaatlichen gerichtlichen Verfahren nichts als ein Verhalten der (Organe bzw. Amtswalter der) Bundesrepublik Deutschland, welches sich im (sachlich-gegenständlich zu verstehenden) Geltungsbereich des Grundgesetzes verwirklicht ${ }^{99}$. Das Auseinanderfallen von völker- und landesrechtlichen Handlungsanweisungen aber ist keineswegs anormal; ein solcher Konflikt wird durch die GGRegeln über das Verhältnis beider Rechtsordnungen zwar verringert, aber nicht immer ausgeschlossen, weder im vertrags- noch im gewohnheitsrechtlichen Bereich ${ }^{100}$.

9. Zu klären bleibt ausschließlich, ob zum Ergreifen vorbeugender Rechtsbehelfe - nach Lage der Dinge vorab im Wege vorläufigen Rechtsschutzes ${ }^{101}$ - alle Bediensteten deutscher Staatsangehörigkeit ${ }^{102}$ oder (darüber hinaus) sämtliche im Bundesgebiet ansässigen Verbandsbeschäftigten ${ }^{103}$ in der Lage sein könnten. Für
Deutsche folgt aus ihrer grundgesetzlich konstituierten Beziehung zu jedwedem Träger deutscher Hoheitsmacht, dem „allgemeinen Gewaltverhältnis“, daß ein verfassungsstaatliches Organhandeln ihnen gegenüber von vornherein nur in begrenztem Rahmen rechtlich zulässig ist, nur (verfassungs-)rechtmäßige Hoheitsakte ihre Position schmälern können ${ }^{104}$. Akte öffentlicher Gewalt zu überprüfen, ist wiederum verfassungskräftig der Judikative zugewiesen, auf deren effektive Kontrolle und Schutz der einzelne Staatsbürger einen subjektiv-öffentlichen Rechtsanspruch hat, wie er zumindest der vollziehenden Gewalt gegenüber in Art. 19 IV GG ausdrücklich festgeschrieben wurde ${ }^{105}$.

Die Antwort auf die zuvor aufgeworfene Frage kann daher nur für die an zweiter Stelle genannte PersonenKategorie zweifelhaft sein, und auch dann nur, wenn es sich bei diesen Individuen um Nicht-Deutsche handelt. Insoweit dürfte nämlich die Grenze dessen erreicht sein, was nach allgemeinem Völkerrecht deutsche Jurisdiktion ausmacht, hier verstanden als internationale Zuständigkeit der Gerichte ratione personae wie materiae ${ }^{106}$. Als territoriale Bezüge zur Staatlichkeit der Bundesrepublik mögen zwar der Aufenthalt eines internationalen Bediensteten im deutschen Hoheitsgebiet sowie die

88 "Legem patere quam ipse fecisti“; so der EuGH in der Rs. 81/72 (Slg. 1973, 575 [583 ff.]); zustimmend H.-P.Ipsen, EuR 8 (1973) 238 (240); Dubouis, R.T.D.E. 9 (1973) 765 (769ff.).

89 Seidl-Hohenveldern, aaO, 1984, 111 (Rdn. 0911), 137 (Rdn. 1102); Meng, aaO, $79 \mathrm{f}$.

90 S. im einzelnen Plantey, aaO, 122 ff.; Amerasinghe, ZaöRV 44 (1984) 439 (454 ff.); ferner A. Weber, ZBR 1978, 326 (331 f.), sowie das EuGH-Erkenntnis in der Rs. 152 ../81 (Slg. 1983, 2357 [2367], mit Schlußanträgen von Rozès, ebd. 2371 [2373])

91 So Art. 101 (3) U.N.-Charta; Langrod, Rev.Héll. Dr. Int. 13 (1960) 97 (115 ff.); Vandersanden, J. D. I. (Clunet) 109 (1982) 660 (672 ff.).

92 Seidl-Hohenveldern, aaO, 140 (Rdn. 1112); Schermers, aaO, I, 159; Hahn, aaO, $106 \mathrm{f}$.; Bowett, The Law of International Institutions, 4. A. 1982, 400.

${ }_{93}$ In bezug auf Art. 31 GG s. BVerfGE 36, 342 (362 ff.); Gubelt, Art. 31, in: von Münch, aaO, II, 299 (303 ff. - Rdn. 14 ff.); Maunz, Art. 31 (1960), in: Maunz Dürig, aaO, Rdn. 13 ff. (5 ff.).

94 S. zum Beispiel die Entscheidung des bayer. VerfGH, BayVBI. 1958, 144 (145 f.), die im zuvor erwähnten BVerfG-Beschluß erwähnt wird (E 36, 360); ferner Maunz, in: Mang/Maunz/Mayer/Obermayer, Staats- und Verwaltungsrecht in Bayern, 4. A. $1975,1(5,25)$.

95 Aus jüngerer Zeit s. etwa BayVerfGH, BayVBl. 1983, 303 (304); BayVBl 1984, 76 (f.). Zu vergleichbaren Anwendungsfragen s. auch Bothe, Fs. Mosler, 1983, 111 (115 ff.).

96 Hierzu für das Recht der E. G. stets noch grundlegend Ipsen, aaO, 1972, 277 und 280; allgemein zum Effektivitätsprinzip bereits H.Krüger, Fs. Spiropoulos, 1957, 265, (278 ff.); s. a. Bernhardt, BerGesVR 12 (1973) 7 (25 ff.); (Neuhold/ Hummer/)Schreuer, aaO, $160 \mathrm{f}$., unter Hinweis auf Zemanek, ebd., 54 (70f.); Brownlie, aaO, $686 \mathrm{ff}$.

97 Vgl. Gramlich, G. Y.I. L. 27 (1984) i. E.; Aubin, Die Haftung der Europäischen Wirtschaftsgemeinschaft und ihrer Mitgliedstaaten bei gemeinschaftsrechtswidrigen nationalen Verwaltungsakten, 1982, $267 \mathrm{ff}$.; Geck, Fs. Carstens, 1984, 339 ( $346 \mathrm{ff}$.)

98 Bothe, Fs. Mosler, $136 \mathrm{f}$. $554 \mathrm{f.})$

Vgl. mutatis mutandis Randelzhofer, Fs. Schlochauer, 1981, 531 (532 und

100 S. Rojahn, aaO, Art. 24/Rdn. 1 a (100); Bermhardt, BVerfG-Fs. II, $182 \mathrm{f}$. zur ähnlichen U.S.-Rechtslage unlängst Henkin, Mich.L.Rev. 82 (1983/1984) $1555 \mathrm{ff}$.

101 So auch (über $\$ 123 \mathrm{VwGO}$ ) in der Sache, die dem 2. „Eurocontrol “ Beschluß des BVerfG zugrundelag (s. E 59, 70 f.). Eingehender zum Verfahren nach \32 BVerfGG Ule, Fs. Maunz, 1981, 395 ff.; E. Klein, AöR 108 (1983) 410 (422 ff.), 561 (616f.).

102 Bei ihnen sollte die von Völkerrechts wegen für nationale (Gerichts-)Zuständigkeit als hinreichend erachtete Binnenbeziehung allein wegen des Nationalitätsbandes gegeben sein (s. Makarov, in: Strupp/Schlochauer (Hrsg.), Wörterbuch des Völkerrechts, Bd. 2, 1961, 129 (130); Wehser, in: Menzel/Ipsen, Völkerrecht, 2. A 1979, 136 [150]). Auch das BVerfG meint offenbar beachten zu müssen, ob insoweit die internationalen Grenzen deutscher Jurisdiktion respektiert werden (s. E 58,$34 ; 59,89$ ).

103 Kritisch zum Kriterium des Ansässigseins Grunsky, Grundlagen des Verfahrensrechts, 2. A. 1974, 341; s. ferner Gramlich, DVBl. 1980, 459.

104 Hesse, Grundzüge des Verfassungsrechts der Bundesrepublik Deutschland, 14. A. 1984, 113 f. (Rdn. 280 ff.). Dies wird nicht gewürdigt von Tomuschat, aaO, Rdn. 13 (18).

105 Zur Reichweite des Art. 19 IV GG insbes. BVerfGE 24, 33 (49 ff.); 35, 382 (401 ff.).

106 Rechtsvergleichender Überblick hierzu bei Steiner/Vagts, Transnational Legal Problems, 2. A. 1976, $729 \mathrm{ff}$. 
Zustimmung zu verbandlichem Wirken angeführt werden, sowohl im Hinblick auf die Organisationsbetätigung überhaupt als auch im Einzelfall in Gestalt der Billigung von im Inland errichteten Anlagen einer Internationalen Organisation ${ }^{107}$. Das Verweilen im Bundesgebiet ist freilich regelmäßig allein dienstlich veranlaßt und typischerweise daher auch nur auf die Zeit der betr. Beschäftigung begrenzt ${ }^{108}$. Der Verband ist dagegen insgesamt gerade von deutscher Gerichtshoheit eximiert ${ }^{109}$. Hieraus dürfte folgen, daß der Umstand der „Residenz“ schwerlich als hinreichend erachtet werden kann, die internationale Gerichtszuständigkeit der Bundesrepublik Deutschland für nichtdeutsche internationale Bedienstete zu bejahen ${ }^{110}$.

10. Die anfechtbare Betätigung des deutschen Staatenvertreters freilich erweist sich in keiner Weise, auch nicht etwa als zusätzlich oder sekundär, als ein Akt des zwischenstaatlichen Verbandes. Sie liegt diesem nicht nur zeitlich voraus ${ }^{111}$, sondern trägt allein beim Zusammentreffen mit anderen, parallelen Willensbildungen und -bekundungen der übrigen mitgliedstaatlichen Repräsentanten zum Entstehen eines der Internationalen Organisation zuzurechnenden Beschlusses bei.

\section{Materielle Maßstäbe}

1. Gewiß wird ein Antrag, wie er vorstehend beschrieben wurde, praktisch wohl nur höchst selten zu einer Verurteilung der Bundesrepublik Deutschland in der Sache führen können. Betrachtet man die Erfolgsaussichten wieder am Beispiel von Verbandseingriffen in die Rechtspositionen internationaler Bediensteter, so erscheint zunächst im vermögensrechtlichen Bereich vom Ergebnis her irrelevant, ob als Maßstab der nationalen Kontrolle auf (Verfassungs-)Rechtmäßigkeit hin auf Elemente des Alimentationsprinzips als einem hergebrachten Grundsatz des Berufsbeamtentums (Art. 33 V GG) ${ }^{112}$ abgehoben wird oder ob - weil diese Bestimmung ja allenfalls dem Sinne nach Anwendung finden könnte ${ }^{113}$ - das Verhalten des deutschen Staatenvertreters an der Eigentumsgarantie des Art. 14 GG $^{114}$ gemessen würde. Im Lichte beider verfassungsrechtlicher Verbürgungen lassen sich nämlich Gehaltskürzungen noch innerhalb der Gestaltungsbefugnisse des legiferierenden Organs ansiedeln ${ }^{115}$. Für eine Rechtsetzung durch internes Staatengemeinschaftsrecht können aber keine strengeren Rechtmäßigkeitsvoraussetzungen gelten, als sie innerhalb eines so grundrechtsbewußten Gemeinwesens wie der Bundesrepublik bestehen ${ }^{116}$. Solange daher die untere Grenze der Bedienstetengehälter so angesetzt bleibt, daß die dem (Verbands-)Beschäftigten verfügbaren Geldmittel zu mehr ausreichen als lediglich zur Befriedigung elementarer menschlicher Grundbedürfnisse nach "Nahrung, Kleidung und Unterhalt "117, erscheint selbst eine nominale Einkommensreduzierung, wenn sie nur alle Gehaltsempfänger gleichmäßig trifft, noch verfassungsrechtlich akzeptabel ${ }^{118}$. Um so mehr dürften Senkungen bloß des Reallohns zumindest dann allein mit politischen Mitteln rückgängig gemacht werden können, wenn der staatliche Mitwirkungsakt innerhalb der Verbands-Willensbildung von einer ordnungsgemäßen Abwägung der wesentlichen Belange getragen wird. Selbst bei Vernachlässigung einiger maßgeblicher Gesichtspunkte jedoch stellt sich die Betätigung nur dann nicht mehr als grundgesetzkonform dar, wenn ihr Inhalt, das Abwägungsergebnis, den von der innerstaatlichen Verfassung eröffneten weiten Rahmen sprengte ${ }^{119}$.
2. Aber auch in bezug auf Meinungs- und Vereinigungsfreiheit, deren Realisierung nicht allein auf staatliche Kommunikationsbeschränkungen stoßen kann ${ }^{120}$, sollte schwerlich Anderes gelten. Der Normbereich der grundrechtlichen Gewährleistungen in Art.5 I, 9 I erweist sich als in internationaler Ausdehnung horizontal modifiziert, einer ihrem Ausmaß nach von der innerstaatlich gebotenen durchaus verschiedenen Regelung als zugänglich. Eine Orientierung an der je grundrechtsfreundlichsten Gesetzgebung der Verbandsmitgliedstaaten $^{121}$ stellt hierbei zwar ein wichtiges außenpolitisches Ziel dar, kann aber nicht alleinige Richtschnur für das

107 Z. B. die Karlsruher Dienststelle von „Eurocontrol“ (Art. 28 des ursprünglichen Übereinkommens 1960, BGBl. 1962 II, 2274, als Rechtsgrundlage für deren Errichtung ist allerdings durch Art. XXII des Änderungsprotokolls 1981 gestrichen worden) oder das Darmstädter "European Space Operations Centre“ der E. S. A. (gem. Art. VI der Gründungskonvention 1975). Für die Zurechnungsfrage zumindest stellt Art. 13 des I. L. C.-Entwurfs über Staatenverantwortlichkeit 1980, Teil 1, klar, der Umstand allein, daß das Verhalten eines Verbandsorgans auf dem Territorium eines Staates stattfand, reiche nicht hin, dies als staatliche Maßnahme zu bewerten.

${ }_{108}$ Nur weil Y.Mortished nach seinem altersbedingten Ausscheiden aus dem Dienst bei den Vereinten Nationen - ihrem Genfer Büro - nicht aus der Schweiz ausreiste, kam es überhaupt zu einem Rechtsstreit über seinen Anspruch auf eine Repatriierungsprämie, da deren Gewährung auch vom Nachweis eines tatsächlichen Wohnsitzwechsels abhing. Gegen die Entscheidung des U. N.-Verwaltungsgerichts (No. 273 vom 30.6.1981, s. A. F.D. I. 27 [1981] 330 [336 ff.]) rief der nach Maßgabe von Art. 11 (1) der verwaltungsgerichtlichen Statuten tätige Ausschuß für Nachprüfungsbegehren auf die Initiative der U.S.A. hin den Internationalen Gerichtshof an. Dieser gab mehrheitlich dem Ausschußantrag auf ein Gutachten statt, konnte aber keine relevanten Rechtsfehler im angefochtenen Erkenntnis ausmachen (I. C. J. Reports 1982, 325 ff.; dazu etwa Tavernier, A. F. D. I. 28 [1982] $392 \mathrm{ff}$; Ruzié, J. D. J. [Clunet] 110 [1983] 76 ff.).

${ }_{109} \mathrm{Vgl}$. bereits Menzel, Fs. Laforet, 1952, 325 (328 ff.); Seidl-Hohenveldern, Fs. Schlochauer, 615 ff.; ders., aaO, 1981, 41 ff., wo auch (45 mit N. 53) auf die E.S. A.-Regelung als eine Bestätigung der tradtionellen Immunitätsbestimmungen hingewiesen wird. In der Tat hebt bereits Art. XV (2) der Gründungskonvention die Existenz von Vorrechten und Befreiungen der Einrichtung selbst wie ihrer Bediensteten hervor und bestimmt sodann Art. IV des Anhangs I, eines integralen Übereinkommensteils (Art. XX [1]), daß die Organisation im Grundsatz Immunität in bezug auf nationale Gerichtsbarkeit an sich wie im Hinblick auf Vollstreckungsmaßnahmen genieße. Allerdings enthält Art. IV (1) (d) des Anhangs I eine Ausnahme im Falle der Beschlagnahme der Gehälter und anderen Leistungen, die die E.S. A. einem Bediensteten schulde, wenn dieser Akt durch eine Entscheidung der (staatlichen) Justizbehörden veranlaßt sei. Diese Vorschrift erlaubt freilich nicht den Austrag von Streitigkeiten zwischen Verband und Funktionär vor mitgliedstaatlichen Richtern, sondern bezieht sich allein auf eine Inanspruchnahme der Organisation als Drittschuldner, bezeichnet also einen generellen, antizipierten Immunitätsverzicht im Hinblick auf Situationen, in denen die Funktionsfähigkeit des Verbands nicht ernstlich beeinträchtigt werden kann.

110 Anderes mag für die Gebiets-Ansãssigkeit im internationalen Wirtschaftsrecht gelten (vgl. Gramlich, aaO, $130 \mathrm{ff}$.). Im Ergebnis wie hier Seidl-Hohenveldern, aaO, 1981, 33, der allerdings dem Status der Beteiligten keine Beachtung schenkt. Demgegenüber hält Bleckmann, aaO, 1981, 25, ein objektives Eigeninteresse der Bundesrepublik für gegeben, daß „auf deutschem Boden belegene Rechtsverhältnisse nach rechtsstaatlichen Prinzipien geordnet und entschieden werden ", ohne jedoch die Radizierung in concreto näher zu begründen (s. a. das Erkenntnis des Berufungsgerichts für den District of Columbia vom 8.1.1980 [Broadbent v. OAS], I. L. M. 19 [1980], 208 [216 f.], unter Bezugnahme auf Akehurst, aaO, 12).

111 Hier drängt sich der Vergleich zur Möglichkeit der nationalen verfassungsgerichtlichen Kontrolle von primärem Verbandsrecht - vermittels des Vertragsgesetzes - auf (s. Maunz, Art. 59 [1961], in: Maunz/Dürig, aaO, Rdn. 15 [10] und 23 [14]).

${ }_{112}$ St. Rspr. seit BVerfGE 8, 1 (16f.); neuerdings etwa BVerfGE 61, 43 (56f.); kritisch Matthey, in: von Münch, aaO, II, 353 (378 - Rdn. 44).

113 Schwerlich hingegen als allgemeiner Rechtsgrundsatz im Verbandsrecht selbst (Baade, A. J. C. L. 15 [1966/1967] 254 [277 ff.]).

114 Insoweit kann von der Parallelitāt beider Gewährleistungen ausgegangen werden (s. BVerfGE 16, 94 [112]).

115 Näher hierzu Gramlich, ZBR 1985, $48 \mathrm{f}$.

116 So plastisch BVerfGE 44, 249 (265).

117 Das stets noch zu beachtende Minimum dürfte jedoch bei Art. 13 des „Europäischen Beamtenstatuts“ (ABI. EG 1968, L 56, 1) unterschritten sein; eine ähnliche, aber sozialstaatsgemäßere Regelung des deutschen Beamtenrechts wurde einhellig für verfassungswidrig erachtet (s. nur Scheffler, in: Bettermann/Nipperdey/Scheuner, Die Grundrechte, IV/1, 1960, 245 [318]).

118 Grundlegend BVerfGE 44, 265 ff.; ebenso etwa Lecheler, AöR 103 (1978) 349 (368ff.); Isensee, in: BendalMaihofer/Vogel (Hrsg.), Handbuch des Verfassungsrechts, 1983/1984, 1149, (1180f.).

119 Insoweit ähnlich Regelungen wie $\$ 44$ a VwGO, $\$ 46$ VwVfG; hierzu jetzt Hill, Jura $1985,61 \mathrm{ff}$.

${ }_{120} \mathrm{Zu}$ Kommunikationsgrundrechten s. Denninger, Staatsrecht 1, 1973, 78 ff.; E. Stein, Staatsrecht, 8. A. 1982, 106; ferner W. Grimm, BayVBI. 1984, 134 (136), sowie BVerfGE 33, 52 (70), und NJW 1985, 121 (122).

121 Vgl. Hailbronner, ZaöRV 36 (1976) 191 (217ff.), insbes. zum EuGHErkenntnis vom 14.5.1974 in der Rs. $4 / 73$ (Slg. 1974, 491 [507f.]); abweichend Bothe, ZaöRV 36 (1976) 280 (290f.). 
organschaftliche Handeln deutscher staatlicher Repräsentanten $\operatorname{sein}^{122}$. Auch dabei zeigt ein Vergleich mit der binnengerichteten Gewährleistung des Grundgesetzes ${ }^{123}$ regelmäßig hinreichend deutlich, was beim Tätigwerden im Rahmen internationalen Organisationsrechts an Grundrechtsminima stets noch zu wahren geboten ist. Mehr Freiheit durchzusetzen wäre möglich, ist aber von Verfassungs wegen nicht erforderlich. Kaum zufällig existieren bislang keine völkervertraglichen Bindungen der zwischenstaatlichen Einrichtungen selbst ${ }^{124}$, kann ein Gebot, bürgerliche, politische, wirtschaftliche oder soziale (Menschen-)Rechte zu respektieren, derzeit bloß aus allgemeinen Rechtsgrundsätzen hergeleitet werden ${ }^{125}$. Nur durch vertragliche Verpflichtungen aber ließe sich ein wahrhaft überstaatlicher, auch in den Internationalen Organisationen verbindlicher Grundrechtsschutz sichern $^{126}$.

122 Ebenso A. Weber, ZBR 1978, 330. Sehr weit reicht allerdings durchweg die Einschränkung der Meinungsfreiheit internationaler Bediensteter (s. Plantey, aaO, $135 \mathrm{ff}$.). Recht typisch hierfür ist E. S. A. Staff Regulation 3.2 und 4.1, wonach sich die Verbandsbeschäftigten ganz allgemein jeglichen öffentlichen Verhaltens, jeder Stellungnahmen oder Publikationen zu enthalten haben, welche mit den Pflichten eines internationalen Amtswalters unvereinbar oder geeignet sind, die moralische oder materielle Verantwortlichkeit der Organisation nach sich zu ziehen. Überdies bedarf eine jede private Verbreitung von dienstlich erlangten, bisher unveröffentlichten Erkenntnissen der Erlaubnis durch den Leiter der Verbandsadministration. $\mathrm{Ob}$ hier nicht bereits die Substanz des Menschenrechts berührt ist, erscheint zumindest erwägenswert (kritisch zu den einschlägigen OECD-Dienstrechtsvorschriften auch Hahn/Weber, aaO, 158 mit N.305; s. demgegenüber freilich die Entscheidung des EuGH vom 17. 5. 1984, DVBI. 1984, 1212 ff.).

123 S. BVerfGE 39, 334 (360), sowie den problematischen Beschluß des Vorprüfungsausschusses zu $\$ 39$ DRiG, NJW 1983, 2691, wo das BVerfG offenbar bestimmte Meinungsäußerungen von vornherein aus dem Schutzbereich des Art. 5 GG ausklammert; vgl. auch Hoffmann-Riem, Art. 5 Abs.1, 2, im AlternativKommentar, aaO, 1, Rdn. 67 f. ( 513 ff.).

${ }_{124}$ S. freilich Sperduti, Fs. Mosler, 1983, $903 \mathrm{ff}$.

125 In diesem Zusammenhang hat sich das Verwaltungsgericht des Europarats neuerdings mehrfach auf Vorschriften der Europäischen Sozialcharta (vom 18.10.1961, BGBl. 1964 II, 1262) bezogen.

126 So auch Ehlermann/Noel, Gs. Sasse, Bd. 2, 1981, 685 (692); s. aber auch Fuss, Der Grundrechtsschutz in den Europäischen Gemeinschaften aus deutscher Sicht, 1975, 78; ders., Gs. Sasse, Bd. 1, 171 (181 und 196); Zuleeg, Fs. Schlochauer, 1981, 983 ( $985 \mathrm{ff}$.).

${ }^{1}$ Vgl. z. B. Hueck/Nipperdey, Lehrbuch des Arbeitsrechts, Bd. I. 7. Aufl. 1963, \$ 44 IV S. $347 \mathrm{ff} . ;$ Nikisch, Arbeitsrecht, Band I, 3. Aufl. 1961, 42 I. II S. $598 \mathrm{ff}$; Söllner, Arbeitsrecht, 6. Aufl. 1978, 32 , 5 S. 224 f.; 8. Aufl. 1984, S. 268 f.; Staudinger/Nipperdey/Mohnen/Neumann, Kommentar zum BGB, 11. Aufl. 1958, \$615 Rdn. 65 ff.; RGRK/Denecke, Kommentar zum BGB, Band II, 10. aufl. 1953, Vorbem. \$ 611 Anm. III, 3.

${ }^{2}$ Nachweise bei Picker, in JZ 1979, 285 (286 Fn.7 und 8).

3 Grundlegend RGZ 106, 272 (275 f.); vom BAG fortgeführt etwa in E 3,346= NJW 1957, 687; 23, $512=$ NJW $1972,600$.

${ }^{4}$ So jetzt das BAG, Beschluß vom 22.12.1980, abgedruckt in NJW 1981, 937.

5 Vgl. z. B. Zöllner, Arbeitsrecht, 3. Aufl. 1983, \$18 V, S. $196 \mathrm{ff}$. m. w. N.

6 Soweit nicht der Mangel auf wirtschaftlichen Schwierigkeiten des Arbeitgebers beruht (sog. Wirtschaftsrisiko). Für diese Fälle wurden schon immer die Regeln des BGB zur Anwendung gebracht.

Vgl. die in Fn. 1 Genannten.

" Vgl. nur Söllner Fn. 1.

4 Vgl. etwa Rückert ZfA 1983, 1 ff.; Picker, JZ 79, 285 ff.; Richardi, JuS 1984, 826 (834 f.); Zöllner a. a. O. Fn. 5; vgl. a. Ernst Wolf, Lehrbuch des Schuldrechts, Zweiter Band: Besonderer Teil, 1978 \$15 D I a S. $198 \mathrm{ff}$.

${ }_{10}$ Nachweise finden sich bei Picker, JZ 1979, 285, Fn. 4.

11 RGZ 106, 272 (275); damals freilich war die Betriebsstörung streikbedingt.

12 ZfA 1983, 1 (3). 